\title{
"Different" as a restriction on Skolem functions*
}

\author{
David Lahm \\ University of Frankfurt
}

\begin{abstract}
An analysis of (a) different as in every child watched a different movie is developed that is based on an analysis of indefinites in terms of Skolemised choice functions. The internal reading of the sentence, which expresses that the movies covary with the children, is analysed as stating that the children can be mapped injectively to movies they watched. This is achieved by letting the Skolemised choice function that interprets the indefinite pick, for each child, an element from the set of movies that the same function does not assign to any other individual, which allows for analysing different in a manner parallelling ordinary intersective adjectives. Simply replacing this set of movies with the set of contextually salient movies then also gives the external reading. An implementation at the syntax-semantics interface is provided, employing Lexical Resource Semantics.
\end{abstract}

Keywords: different, choice functions, Skolem functions, indefinites, Lexical Resource Semantics

\section{Introduction}

The adjective different is known to have what Carlson (1987) calls an external and an internal reading: (1) is ambiguous between (2a), where the context determines what the movies watched are said to be different from (the external reading), and $(2 b)$, where this information comes from the sentence itself (the internal reading).

(1) Every child watched a different movie

(2) a. Every child watched a movie not among the contextually salient ones.

b. Every child watched a movie distinct from those the other children watched.

Brasoveanu (2011) gives an account of the semantics of different that offers a unified analysis of the external and internal readings. But, as pointed out by Bumford \& Barker (2013), his analysis does not extend to cases in which different finds multiple every-or each-phrases that could serve as its antecedents, as in (3).

* Thanks to Frank Richter, Manfred Sailer, Ede Zimmermann and Cécile Meier for support and discussions and to the audience at SALT 26 for valuable discussion. The research reported here was funded by the German Research Foundation (DFG) under grant GRK 2016/1.

C) 2016 Lahm 
"Different" as a restriction on Skolem functions

(3) Every girl thinks that every boy watched a different movie starring his mother.

(3) is ambiguous, depending on which NP different selects as its antecedent. With every girl as antecedent it can mean that there is a different movie for every girl which stars every boy's mother and which the girl believes every boy to have watched. But under Brasoveanu's account, the covariance expressed by different always has to be with the scopally closest antecedent. Bumford and Barker offer a modification of Brasoveanu's analysis which they claim predicts the correct readings for sentences like (3), but this claim will be seen to be incorrect in section 2.3.

The analysis proposed here concentrates on (a) different $N$ with every or each as the antecedent quantifier. It adopts an analysis of indefinites in terms of centered Partial Skolemised Choice Functions (centered PSCFs). As in Kratzer 1998, choice functions can be Skolemised, ${ }^{1}$ which makes it possible to model scopal relations functionally. Differently from Kratzer 1998, the function variables are not assumed to remain free but to be bound by existential quantifiers, which will be seen to have welcome effects. The analysis of different then has the adjective restrict the possible Skolemised choice functions in such a way that a sentence like (1) comes out as true iff there is an injective function that maps children to movies they watched. This restriction will be spelt out by letting different require that the movie each child is assigned by the function be not in the set of objects any other individual is assigned, similarly to how American in American movie requires the movie to be in the set of American things. This allows for closely assimilating the analysis of different to that of intersective adjectives, from which it is only distinguished in that the pertinent set depends on a variable bound by every or each. The external reading is arrived at by replacing this dependent set with the set of contextually salient movies.

The paper is organised as follows. In section 2, I briefly discuss previous work on different by Beck (2000), Brasoveanu (2011) and Bumford \& Barker (2013). Section 3 contains the analysis. It introduces centered PSCFs and shows their use in the analysis of different and further discusses sentences with multiple antecedents and intensional contexts. Section 4 implements the analysis at the syntax-semantics interface, using Lexical Resource Semantics, a variety of underspecified semantics, and section 5 solves certain problems with existential quantification over PSCFs.

1 The possibility of analysing different in terms of Skolemised choice functions has already been pointed out in Kratzer 1998:

A different [is] 'perspectival' in the sense of Mitchell and Partee. [It has] implicit arguments that can have bound variable interpretations. For [every professor will rejoice if a different student cheats on the exam], the bound variable interpretation implies that for different professors $x$ and $y$, we have different choice functions $f_{x}$ and $f_{y}$ that pick out different students." (Kratzer 1998: 169) 
Lahm

\section{Previous approaches}

\subsection{Beck 2000}

Beck (2000) argues that there is a fundamental difference between different depending on plural NPs, exemplified in (4), and depending on morphologically singular quantifiers, exemplified in (1). The claim is motivated by the fact that German employs distinct lexical items in these cases, as shown in (5a) and (5b).

(4) Ede and Wolfgang watched different movies.

a. Jeder Professor hat einen anderen Film gesehen. every professor has a different movie seen

b. Ede und Wolfgang haben verschiedene Filme gesehen. Ede and Wolfgang have different movies seen

Beck argues that verschieden is a relational adjective and that its meaning in a sentence like (5b) is intimately tied to plural semantics, while ander- is a comparison operator whose semantics is related to that of the comparative. In this paper, I also assume, tentatively, that the plural-dependent and the singular-dependent reading demand different analyses, but without committing myself to Beck's claims about the nature and depth of this difference. In particular, for the time being, I follow Beck in assuming that the internal reading of different occuring in a singular NP requires a morphologically singular antecedent. ${ }^{2}$ I am here only concerned with this case, i.e. different depending on one of the quantifiers every or each.

Beck's semantics for the internal reading with Q-bound different assumes a semantics that requires the universal quantifier to bind two distinct variables but is silent on how this is to be derived. In the next section, I shall briefly discuss how Brasoveanu (2011) implements a variant of this idea. The proposal advocated here can do without this binding of two variables.

\subsection{Brasoveanu 2011}

Brasoveanu (2011) offers a unified treatment of the external and internal readings of different. His account of the internal reading relies on a dynamic semantic system

2 Frank Richter (p.c.) doubts the validity of this generalisation. These doubts are provisionally supported by an example found in the COW14 corpus of English (cf. Schäfer 2015), a good leader will understand that all team members have a different way of viewing things, which only makes sense on an internal reading and was quickly found. Determining how common and felicitous such examples actually are must be left to future research. Also, especially in colloquial German, anderand verschieden often appear in contexts in which, according to Beck, only their counterparts should be allowed. Even if one should find evidence to regard these cases as systematic errors, it would still remain an interesting question why such errors can occur with a notable frequency if the two items have very distinct semantic properties. 
"Different" as a restriction on Skolem functions

and the assumption that quantifiers like every and each employ an operator Dist to distribute their scopal content over the atoms of their restrictor sets. This operator also opens up a second information state in addition to the primary one to which the meanings of most other expressions solely contribute. This information state makes available during the update with Dist for each element $x$ of the restrictor the result of the corresponding update for all elements of the restrictor distinct from $x$. In this context, different can then require the non-identity of the movies which have been introduced in the two distinct information states. By allowing different to relate to previously introduced movies instead of those introduced in the course of the update, the external reading is also explained. In effect, Brasoveanu derives (6) as the meaning of the internal reading of (1).

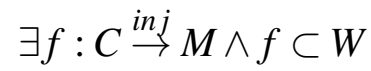

I.e. there is an injective function taking children to movies which is a subset of the watch relation (assuming the typical set-theoretic analysis of relations as sets of ordered pairs and functions as relations). ${ }^{3}$

This is the meaning I shall also assume, but I shall make the assignment of children to movies explicit in the form of a Skolemised choice function. This will solve the problem with multiple antecedents discussed in the following section.

\subsection{Bumford \& Barker 2013}

Bumford \& Barker (2013) point out that Brasoveanu's account encounters difficulties where a sentence contains multiple possible antecedents for different. Of particular relevance for the argument are examples of the type shown in (7).

(7) Every girl thinks that every boy watched a different movie starring his mother.

This sentence has a reading under which there is, for each girl, a movie starring the each boy's mother which the girl believes every boy to have watched. In (7), Bumford \& Barker (2013) argue, the scope order needs to be $\forall_{\text {girl }}>$ think $>\forall_{\text {boy }}>$ $\exists_{\text {movie }}$ because $\forall_{\text {boy }}$ is confined to the that-clause and binds a variable in the restrictor of $\exists_{\text {movie }}$. Hence different cannot pick $\forall_{\text {girl }}$ as its antecedent because only the scopally lowest operator can act as an antecedent under Brasoveanu's account.

The solution that Bumford \& Barker (2013) propose has every each- and every-phrase introduce an additional stack $^{4}$ which in a manner roughly paralleling

3 This is one of the readings that Keenan (1992) considers, ascribing it to van Benthem (1989). The latter however may have intended an even stronger version, requiring $f=(C \times M) \cap W$. At the very least, (6) expresses a necessary condition for the truth of (1). Discussing whether it should be regarded as sufficient must be left to future work.

4 Stacks (basically, partial variable assignments) are also employed by Brasoveanu, but the plural info states he works with are sets of stacks, not the single stacks Bumford and Barker replace them 
Brasoveanu's analysis is supposed to mediate the needed information. But what Bumford \& Barker's (2013) account actually derives as the meaning of (7), assuming that a different movie starring his mother is supposed to covary with the girls, is approximately what is shown in (8).

$$
\begin{aligned}
& \forall x y(x \neq y \wedge x, y \in G \rightarrow \\
& \quad x, y \in T H(\lambda w . \forall z(z \in B \rightarrow \exists u v(u \neq v \wedge u, v \in P(z) \wedge R(z, u) \wedge R(z, v)))))
\end{aligned}
$$

But this simply ascribes to every girl the belief that every boy watched at least two movies starring his mother, which is not a possible reading of (7). To arrive at the intended reading, the indefinite would somehow need to take scope outside of the that-clause, something that (7) was expressly designed not to allow. ${ }^{5}$ The problem of the analysis is that the variable bound by every girl does not occur bound anywhere in the embedded proposition and hence cannot play any semantic role. ${ }^{6}$ This will be different under the present approach, where the variable will appear as an argument to a Skolemised choice function.

\section{Analysis}

\subsection{Centered Partial Skolemized Choice Functions}

The present account of the phenomenon rests on an analysis of indefinites by means of centered Partial Skolemised Choice Functions (PSCFs). Like ordinary choice functions, these take a set as an argument and their ultimate entity-type value needs to be an element of this set argument. In addition, they can take an arbitrary number of individuals as further arguments. Filling these argument slots with variables bound by quantifiers, PSCFs can be used to model scopal dependencies on quantifiers that are actually outscoped by the quantifier that binds the function variable.

While Skolem functions and choice functions are well established in semantics (e.g. Reinhart 1997; Winter 2001: §3; Kratzer 1998), the concept of Centering is, to my knowledge, new. It solves some of problems pointed out by Geurts (2000).

with. As a consequence of (inter alia) this, they would have to assume, unlike Brasoveanu, that the indefinite article means "exactly one". They do not explicitly make this assumption, but without it (1) is predicted to be true if 100 children each watched the same two movies, an unwelcome result.

5 The indefinite could be allowed though to take scope outside of the that clause due to a specificity effect (which is what basically happens under the present account, too, but is analysed here using the choice function approach). The problematic anaphor in the restrictor could then remain bound if the restrictor could be stranded, perhaps along the lines in Szabó 2010. But choosing this route would clearly put Brasoveanu's approach back in the race, and under Bumford \& Barker's (2013) account, reading (8) still would need to be ruled out.

6 By the coincidence lemma. 
"Different" as a restriction on Skolem functions

\section{Choice Functions}

A (proper) choice function is a function that takes a set as its only argument and whose value for any argument always is an element of that argument.

Definition 1 (Choice Function) A function of type $f_{\langle\langle\tau, t\rangle, \tau\rangle}$ is a Choice Function iff for every $S \in D_{\langle\tau, t\rangle}$, if $S \neq \emptyset$, then $S(f(S))$.

The condition $S \neq \emptyset$ in definition 1 raises the question what $f(\emptyset)$ should be. It cannot be an arbitrary object, since then John saw a unicorn would mean John saw something if there are no unicorns (cf. Geurts 2000). Thus choice functions are here conceived of as partial and thus undefined for (at least) the empty set.

\section{Partial Skolemised Choice Functions}

A Partial Skolemised Choice Function (PSCF) takes a set $S$ as its first argument (the set argument) and any number of entities as further arguments (the entity arguments) and ultimately returns a member of $S$, if anything. I allow these functions to be undefined not only for the empty set argument but also for non-empty set arguments and entity arguments. Allowing undefinedness for non-empty set arguments will be crucial to allow for Centering. Allowing undefindeness for entity arguments is necessary to get the right predictions in certain special cases.

Following Reinhart (1997), function variables introduced by indefinites are bound by existential quantifiers which can freely choose where to take scope. So Every student read a book receives the following possible analyses with a widescope reading (9a) and narrow-scope reading (9b). A second conceivable narrowscope reading is $(9 \mathrm{c})$, which also illustrates Skolemisation: the value of $f(B)(x)$ functionally depends on $x$. (9c) will be ruled out however by the analysis developed below, which assumes that only items like different license Skolemisation. ${ }^{7}$

$$
\begin{aligned}
& \text { a. } \exists f(P S C F(f) \wedge \forall x(S(x) \rightarrow R(x, f(B)))) \\
& \text { b. } \forall x(S(x) \rightarrow \exists f(P S C F(f) \wedge R(x, f(B)))) \\
& \text { c. } \exists f(P S C F(f) \wedge \forall x(S(x) \rightarrow R(x, f(B)(x))))
\end{aligned}
$$

7 This assumption is not essential. It only serves to rule out the overrepresentation that comes with having both (9b) and (9c). But overrepresentation (i.e. licensing of syntactically distinct but logically equivalent logical forms) seems a purely aesthetic problem. Yet a possible benefit of restricting Skolemisation to cases in which it is really needed is that Skolem functions might be regarded as too powerful to be generally allowed, as an anonymous reviewer pointed out. E.g., choice functions can be used to express branching quantification, whose existence in natural language is by far not universally acknowledged. 
Since partial functions will be needed, $f(B)(x)$, for instance, might be undefined for certain values of $x$. This will lead to undefinedness of $R(x, f(B)(x))$ and generally all atomic formulae it occurs in. Undefined truth values are dealt with along the lines of Kleene's strong three-valued logic, except for the existential quantifier $\exists f$, which for the moment can be assumed to be false iff $\phi$ is not true for any value of $f{ }^{8}$

\section{Centered PSCFs}

Centered PSCFs can now be defined as in definition 2.

Definition 2 (Centered PSCF) A PSCF $f$ is centered iff there is a partial function $c$ such that for any $S$ and any vector of entities $\vec{x}: c(\vec{x})$ is defined if $f(S)(\vec{x})$ is and, if both are defined, $f(S)(\vec{x})=c(\vec{x})$. Call $c$ the center of $f, C t(f)$.

If a PSCF is centered, the set argument no more plays any role in determining its final result apart from specifying a set it needs to be contained in. ${ }^{9}$ Together with the way undefined values are handled, this closely assimilates quantification over non-Skolemised choice functions to quantification over individuals: such functions are constant where defined. I believe centering to solve most of the problems with choice-functional analyses discussed by Geurts (2000). Henceforth existential quantifiers are implicitly assumed to range over centered PSCFs.

\section{2 "Different" as a restriction on centered PSCFs}

We can now assign to a sentence like every child watched a different movie the following representation, which will be abbreviated as (10b), adopting the notation for Skolemised choice functions from Kratzer 1998.

$$
\begin{aligned}
& \text { a. } \exists f \forall x\left(C(x) \rightarrow W\left(x, f\left(\lambda y \cdot M(y) \wedge \neg \operatorname{Im}^{-}(f)(x)(y)\right)(x)\right)\right) \\
& \text { b. } \exists f \forall x\left(C(x) \rightarrow W\left(x, f_{x}\left(\lambda y \cdot M(y) \wedge \neg \operatorname{Im}_{f}^{-x}(y)\right)\right)\right)
\end{aligned}
$$

The family of constants $\mathrm{Im}^{-}$is defined as exemplified in definition 3, where it is defined for PSCFs with a single individual argument.

Definition 3 (Except-for-Image) The image of a Centered PSCF $f$ except for $x$ is the set

$$
\operatorname{Im}^{-}(f)(x):=\lambda z . \exists y: x \neq y \wedge \uparrow C t(f)(y)=z
$$

8 This is too simplistic an assumption and will be revised in section 5.

9 It is an immediate consequence that $f(S)(x)$ and $f\left(S^{\prime}\right)(x)$ cannot both be defined if $S$ and $S^{\prime}$ are disjoint; extending partiality to non-empty sets is thus essential for centering to be possible at all. 
"Different" as a restriction on Skolem functions

of all entities that can be values of $f$ for arguments other than $x$. $\uparrow t$ means that $t$ is defined. There is no constant $\mathrm{Im}^{-}$for $f$ of a pure choice function type, i.e. without individual arguments.

So what (10a) says is that there is some function $f$ such that, for every child $x, x$ watched $f_{x}\left(\lambda y \cdot M(y) \wedge \neg m_{f}^{-x}(y)\right)$. Since $f$ needs to take a value from its set argument, this value needs to be a movie that $f$ assigns to no other individual but $x$. Since every child is thus assigned a movie that nothing else is assigned by $f$, the restriction of $f$ to $C$ will be an injective function, just as desired.

Note that the 'basic' contribution of different is $\lambda y . \neg m_{f}^{-x}(y)$, which is combined with the contribution of the noun in exactly the same way as in the case of intersective adjectives, as illustrated in (11a) (different ball) and (11b) (red ball).
a. $\lambda y \cdot B(y) \wedge \neg \operatorname{Im}_{f}^{-x}(y)$
b. $\lambda y \cdot B(y) \wedge R(y)$

Where different deviates from intersective adjectives is thus not its local combinatoric behaviour but the fact that it can allow for variables to be bound by the quantifiers every and each.

\subsection{Multiple antecedents}

The analysis of different just outlined does not encounter the problems with multiple possible antecedents for different-containing NPs that Brasoveanu's account did. A sentence like (12) can receive the analyses in (13).

(12) Every girl thinks that every boy watched a different movie starring his mother.
a. $\exists f \forall x\left(G(x) \rightarrow T H\left(x, \wedge \forall y\left(B(y) \rightarrow R\left(y, f_{x}\left(\lambda z \cdot M(y)(z) \wedge \neg \operatorname{Im}_{f}^{-x}(z)\right)\right)\right)\right)\right)$
b. $\exists f \forall x\left(G(x) \rightarrow T H\left(x, \wedge \forall y\left(B(y) \rightarrow R\left(y, f_{y}\left(\lambda z \cdot M(y)(y) \wedge \neg \operatorname{Im}_{f}^{-y}(z)\right)\right)\right)\right)\right)$
c. $\forall x\left(G(x) \rightarrow \exists f T H\left(x, \wedge \forall y\left(B(y) \rightarrow R\left(y, f_{y}\left(\lambda z \cdot M(y)(y) \wedge \neg \operatorname{Im}_{f}^{-y}(z)\right)\right)\right)\right)\right)$
d. $\forall x\left(G(x) \rightarrow T H\left(x, \wedge \exists f \forall y\left(B(y) \rightarrow R\left(y, f_{y}\left(\lambda z \cdot M(y)(y) \wedge \neg \operatorname{Im}_{f}^{-y}(z)\right)\right)\right)\right)\right)$

Reading (13a) expresses that the girls can injectively be mapped to individuals in such a way that each girl believes that every boy watched the object the girl is assigned and that this object is a movie starring the boy's mother. So for each girl there is a different movie which she believes every boy to have watched. Figure 1(a) illustrates a situation that verifies this reading.

Reading (13b) expresses that the boys can injectively be mapped to individuals in such a way that each girl believes that every boy watched the object he is mapped 


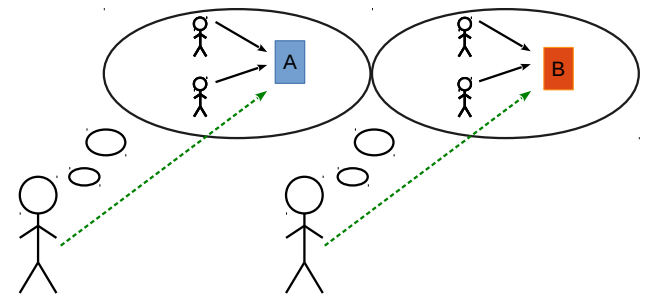

(a) Variation with the girls

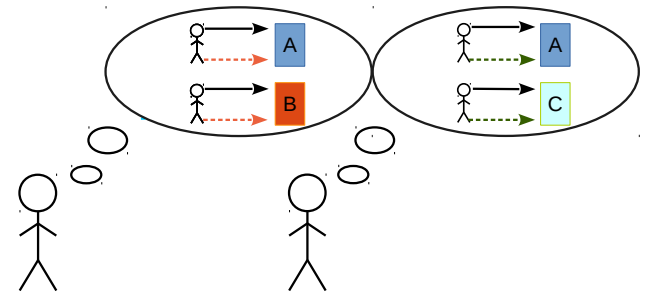

(c) Variation with the boys and possibly the girls

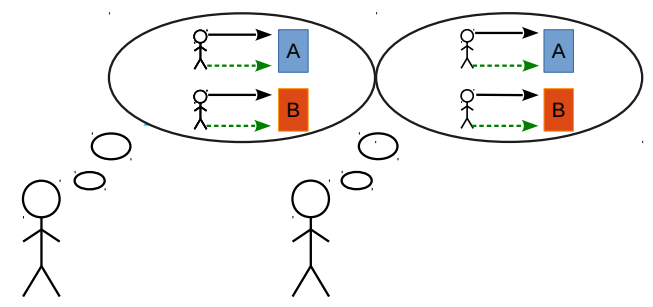

(b) Variation with the boys

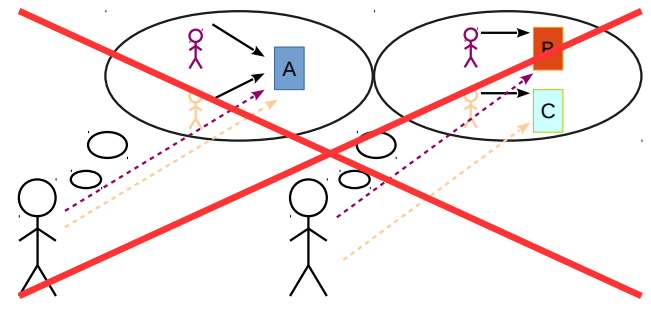

(d) Variation with the girls and possibly the boys (ruled out)

Figure 1 Illustrations of different readings of (12).

to and that this object is a movie starring his mother. Since $\exists f$ takes widest scope, there must be a movie for each boy that each girl believes him to have watched. An illustration is provided in Figure 1(b). Reading (13c) expresses that a function can be found for each girl that injectively assigns movies to boys such that the girl believes each boy to have watched the movie assigned to him. Since $\exists f$ takes scope now below every girl, the movies may, but of course need not, vary with the girls, too. Since the variation is optional, both 1(b) and 1(c) verify this reading.

Reading (13d) expresses that each girl believes that movies watched can be assigned to boys injectively. This reading is also verfied by $1(\mathrm{~b})$ and $1(\mathrm{c})$, but since it is de dicto, what the movies $A, B$ and $C$ may vary with the different worlds in the girls' doxastic states. In the preceding cases, $A, B$ and $C$ each need to stand for the same movie in all of the doxastically accessible worlds of each girl. ${ }^{10}$

10 Whether all of these readings should be regarded as existent in their own right is a difficult question as they are not all independent. E.g. (13c) follows from (13b). But it seems that none of these readings is definitely uavailable. 
"Different" as a restriction on Skolem functions

\begin{tabular}{l|l|l} 
owe & Matt & Ben \\
\hline Crook & 2000 & 3000 \\
Lowlife & 2000 & 4000
\end{tabular}

Table 1 A situation that verifies (14a): for every loan shark an injective mapping from actors to sums they owe him exists.

That every boy should not normally be able to take scope outside of the thatclause is expected to restrict the possible readings of (12), in contrast to those of sentences without embedded clauses. This prediction is borne out. Consider sentence (14a): the sentence is similar to (12) in containing two potential antecedents for different but differs from it in that both of these are constituents of the same clause. For (14a), the reading (14b) is correctly predicted. Table 1 provides illustration (where Matt and Ben are all the actors and Crook and Lowlife all the loan sharks).

a. Every actor owes every loan shark a different sum.

b. $\forall x\left(L(x) \rightarrow \exists f \forall y\left(A(y) \rightarrow O\left(y, x, f_{y}\left(\lambda z . S(z) \wedge \neg \operatorname{Im}_{f}^{-y}(z)\right)\right)\right)\right)$

But the structurally corresponding reading represented in (15) is predicted to be unavailable for (12), as it would require every boy to take widest possible scope while this quantifier should be confined to the that-clause (or its vicinity). Figure (d) shows a situation that would verify this reading but does in fact not seem to verify any actual reading of (12).

$$
\begin{aligned}
* \forall x(B(x) & \rightarrow \\
& \exists f \forall y(G(y) \rightarrow \\
& \left.\left.\rightarrow H\left(y, W\left(x, f_{y}\left(\lambda z \cdot M(x)(z) \wedge \neg \operatorname{Im}_{f}^{-y}(z)\right)\right)\right)\right)\right)
\end{aligned}
$$

I conclude this section with a remark on simultaneous antecedents: since PSCFs may take more than one individual argument, reading (16) is predicted to exist for (14a), where every actor and every loan shark act as antecedents simultaneously.

$$
\exists f \forall x\left(A(x) \rightarrow \forall y\left(L(y) \rightarrow O\left(x, y, f_{x, y}\left(\lambda z . S(z) \wedge \neg \operatorname{Im}_{f}^{-x, y}(z)\right)\right)\right)\right)
$$

I.e. each pair of an actor and a loan shark is assigned a different sum. (16) implies both of the readings in which $\exists f$ has narrow scope with respect to one of the universal quantifiers. While it is very salient at times, it is thus not trivial to tell whether it should actually be regarded as a reading in its own right, but the present account provides for that possibility. ${ }^{11}$

11 Cf. footnote 10 


\subsection{The external reading}

Given the system developed above, accounting for the external reading of the sentences in question is merely a matter of replacing the $\mathrm{Im}^{-}$expression with an expression $\operatorname{Sal}(X)$ which is interpreted as the set of contextually salient $X$. So for (1), i.e. every child watched a different movie, the representation will be (17).

$$
\exists f \forall x(C(x) \rightarrow W(x, f(\lambda y \cdot M(y) \wedge \neg \operatorname{Sal}(M)(y))))
$$

Different is lexically specified to employ either a Sal or an $\mathrm{Im}^{-}$expression. In a context like the teacher recommended Taxi Driver and Heat, but ..., Sal(M) will be $\{$ Taxi Driver,Heat $\}$ and (17) will express that there is a movie that is neither Taxi Driver nor Heat which every child watched. $\exists f$ also may take scope under $\forall x$, allowing the movies to vary with the children. These seem to be the correct results.

\subsection{Remarks on intensional contexts}

In (13), the question of the precise locus of evaluation for the non-logical constants has so far been glossed over. The presentation suggests that everything embedded underneath ${ }^{\wedge}$ is to be evaluated inside of the pertinent girl's doxastic state. Since this is possible - but not necessary - the account admits a variant of restrictor stranding as advocated for by Szabó (2010). ${ }^{12}$ But under our account, the restrictor always remains in situ while Szabó (2010) does only allow but not require stranding.

But this does not mean that the restrictor must be evaluated inside of the doxastic state. As argued by Bäuerle (1983), the restrictors of quantifiers can quite generally be interpreted outside of their embedding contexts, and it seems plausible that this also holds for the set arguments of choice functions. Hence the beliefs of the girls in (13) could well be about actual boys, without any claim that the girls believe them to be that. But importantly, this does not have to be the case: if Mary mistakes a group of girls for a group of boys and forms the right beliefs about them, she might well be said to believe that every boy watched a different movie starring his mother with the indefinite interpreted de re, even though her belief is actually about girls.

Note that if such a sentence is to be about individuals only believed by Mary to be boys, the extension of $B$ can vary with the worlds in Mary's doxastic state. It is easily seen that the function $f$ involved in the interpretation of $a$ boy ... will then be injective if restricted to the whole of $\left\{a \mid\right.$ for some $w \in D_{\text {ox }}$ Mary,$\left.a \in B_{w}\right\}$, the set of individuals that Mary does not believe to be no boys. This seems to be the right result: assume that Mary has firm beliefs regarding who in a group of children has seen which movie. Further assume that, while she is not sure regarding some

12 This is, of course, only true so far for indefinites, while Szabo claims the scope of the phenomenon to be much larger. 
"Different" as a restriction on Skolem functions

children whether they are boys, girls or neither of both, no matter what the set of boys is in a particular world in her doxastic state, there always is the required kind of injective mapping to watched movies. It seems justified then to say (intending the de re interpretation) that Mary thinks that every boy watched a different movie.

But there are problematic cases, too. Assume that Mary expects her brother's family. Her brother has four children, two pairs of non-identical twins, where both pairs consist of a boy and a girl and the pairs are a few years apart. Now Mary believes de re that the boys will each recite a different poem, namely that the younger one will recite Jabberwocky and the older one The Rime of the Ancient Mariner. But when Mary sees the children approaching, she first cannot discern who the boys and who the girls are but only who the older and who the younger children are. So the leftmost child, assume him or her to be among the older ones, could be the older boy or the older girl and similarly for the others, which leaves four different configurations. But in each world in Mary's doxastic state, no matter which of these configurations is realised, the younger boy should be about to recite Jabberwocky and the older boy to recite The Rime of the Ancient Mariner. Then there clearly is no injection of the required kind, i.e. with domain $\left\{a \mid\right.$ for some $w \in D_{\text {ox }}$ Mary,$\left.a \in B_{w}\right\}$, which contains four individuals that must be mapped to only two poems.

While this is certainly a problem for the system as it is presented here, it is far from clear whether it should be regarded as a problem of analysing different along the lines proposed or rather as a problem of analysing de re quantification as quantification over individuals. Adjusting the present theory to an analysis of de re quantification in terms of quantification over individual concepts might provide a satisfactory solution to the problem and is called for on independent grounds (cf. Aloni 2001: $\$ 2$ and references there), ${ }^{13}$ but must be left to future work.

\section{Syntax-semantics interface}

This section introduces the syntax-semantics interface that assigns the appropriate meanings to sentences involving different. The most obvious question the present account raises is how the arguments to the PSCFs end up in the right places and are bound in the right way in expressions like (18).

$$
f_{x}\left(\lambda y \cdot M(y) \wedge \neg m_{f}^{-x}(y)\right)
$$

13 Another interesting case is the following: suppose Mary believes that Bill met the president of the George Clooney fan club and that John met the vice president of the Jack Nicholson fan club. She suspects that these might in fact be the same person, but is not committed to this idea. In fact, they are not. It seems that Mary believes that every boy/each of Bill and John met a different person can felicitously and truthfully be uttered in this situation, but it is neither true de dicto nor de re if de re quantification is analysed as quantification over individuals. 
Three things about (18) are noteworthy: (i) the two occurrences of the variable $x$ need always be occurrences of the same variable, (ii) these need to be bound by one of the quantifiers every and each and (iii), the variable $f$ needs to occur as an argument to $\mathrm{Im}^{-}$in addition to being the outer functor.

\subsection{Lexical Resource Semantics}

These issues are resolved here by employing Lexical Resource Semantics (LRS). LRS uses the model theory developed for HPSG grammars by King (1989) and Richter (2004) to employ techniques from underspecified semantics while at the same time predicting fully specified semantic representations for each linguistic object. In this section, I can only give a brief sketch of the system. For a more thorough introduction, see Iordăchioaia \& Richter 2015: §3.2. For a crisp and formally fully explicit statement of the system see Penn \& Richter 2004: 2 .

In LRS, the composition of meaning is not modelled via a syntactic stratum like LF and with functional application as the primary means of obtaining the meanings of complex expressions from their parts, but by constraining the way in which expressions of a formal language ${ }^{14}$ that are contributed by the lexical items in an expression can be put together. This is done by constraining the ways in which these expressions may or may not occur as subexpressions of each other.

The semantic contribution of each syntactic object (sign) is the value of the attribute LRS and split into three distinct objects, the values of the attributes INCONT, EXCONT and PARTS. The first two take meaningful expressions as their values while the last one takes a list of meaningful expressions.

The PARTS list of a word is specified in the lexicon and contains the semantic material the word contributes to the meaning of the sentence. When a complex sign is formed, its parts are the union of the parts of its daughters. Thus every part of the semantics must stem from a lexical item. In an unembedded sign (an utterance), all and only these accumulated parts must be used, i.e. occur as subexpressions of the sign's meaning representation, which is identified with its EXCONT. The INCONT value of an expression is typically the scopally lowest expression that the head of the sign contributes and the EXCONT value typically represents the contribution of the maximal projection of the sign.

These three parts are related by constraints enforcing that the INCONT always be a subexpression of the EXCONT (and hence always must contribute to the meaning of the sign's maximal projection) and an element of the PARTS list. INCONT and EXCONT values of complex signs are always projected from the syntactic head.

How the contributions of the daughters of complex signs have to be combined

14 Typically, two-sorted type theory, which is also assumed here, but in a variant extendend with partial functions. 
"Different" as a restriction on Skolem functions

is determined by the construction-specific clauses of the SEMANTICS PRINCIPLE (SP). For the combination of an $\mathrm{N}$ projection with a quantificational determiner, the principle requires the INCONT of the noun to be a subexpression of the restrictor of the quantifier contributed by the determiner. Since indefinites are analysed using choice functions, the set arguments of these functions also count as restrictors in this sense. Furthermore, the EXCONT value of the head daughter (i.e. the nominal projection) is required to be identical with that of the non-head daughter (the determiner). Combining an NP with a verbal projection requires the INCONT of the $\mathrm{V}$ to be a subexpression of the scope of the quantifier that is the NP's EXCONT, if the EXCONT actually is a quantifier. If it is a choice function expression $f(\lambda x . \phi)$, the SP has nothing to say about it, but lexical argument linking will ensure that the expression occurs as an argument in the appropriate place.

\subsection{The analysis of "different"}

Mainly two things are needed to implement the present analysis of different in LRS: a lexical entry for the indefinite article that is adapted to the PSCF analysis and a lexical entry for different itself. I turn to the indefinite article first. ${ }^{15}$

As the internal content of the indefinite article, an expression of the form $f(\lambda x . \phi)$ seems a natural choice, where $f$ should of course be a variable ranging over centered PSCFs and $\lambda x . \phi$ should be the restrictor of the indefinite NP. Regarding the EXCONT, there are two possible options: either it is identical with the INCONT or it also comprises the quantifier $\exists f$. The first of these options is chosen here as it allows for an easier and general formulation of the SP clause that manages Det-N constructions. Additionally, this choice can be regarded as an analogue of the idea of freely applicable existential binding of choice function variables proposed by Reinhart (1997). ${ }^{16}$ These considerations lead to the partial lexical entry in (19). ${ }^{17}$

15 The analysis of the indefinite article is based on an as yet unpublished analysis of the definite article in terms of the 1 -operator from Levine, Richter \& Sailer (in preparation), due to Manfred Sailer.

16 While this mechanism is not available in its 'pure' form unless the assumption that every part of the meaning representation of an utterance must have been lexically contributed is dropped, it can be approximated by including the existential quantifier on the PARTS list of the indefinite article while not treating it as a part of the external content.

17 Tags like 1, a familiar component of the HPSG notation, are variables that signify token-identiy. For example, according to the present lexical entry, the INCONT and EXCONT of the indefinite article always are the very same object. Tags do thus not belong to the object language Ty2. The notation $\alpha[\beta]$ signals that $\alpha$ contains $\beta$ as a subexpression and $[\alpha]$ may be used to refer to any expression containing $\alpha$ as a subexpression. 


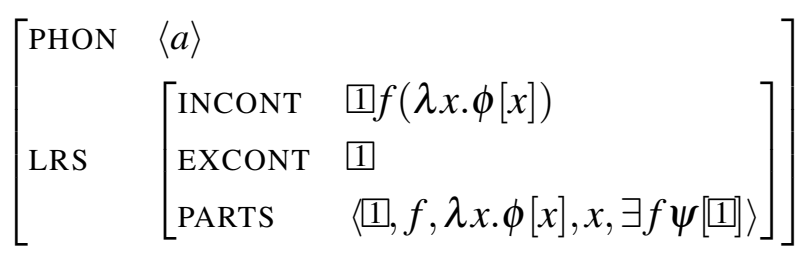

To restrict the antecedents of a different $N$ to every and each, ${ }^{18}$ I further assume the lexical entry to constrain $\psi$ so as to allow occurrences of $f(\lambda x . \phi[x])$ therein to only (if at all) be applied to variables bound by an occurrence of $\forall$ inside of $\psi$. Under the assumption that only every and each contribute this first order quantifier while its plural counterpart all receives a distinct plural semantic analysis, this predicts that covariation of a different-NPs is only possible with those two quantifiers.

It has already been remarked that the analysis of different suggested here assimilates the combinatoric behaviour of this adjective to that of intersective adjectives. The treatment of these I adopt from Sailer 2004: an intersective adjective like red has $R(x)$ as its INCONT and $(\phi[x] \wedge R(x))$ as its EXCONT. The SP requires the INCONT of the modified head daughter to be a subexpression of $\phi$, so the relevant aspects of the contribution of red car can be summed up as $(\phi[C(x)] \wedge R(x))$ (where typically $\phi \equiv C(x)$ will hold).

Now a partial lexical entry for different can be stated as in (20).

$$
\left[\begin{array}{lll}
\text { PHON } & \langle\text { different }\rangle \\
\text { LRS } & {\left[\begin{array}{ll}
\text { INCONT } & 1 \neg F[3(y) \\
\text { EXCONT } & 2(\phi[y] \wedge 1) \\
\text { PARTS } & \langle 1, F, 2, f(\lambda y .2)(3, \cdots\rangle
\end{array}\right]}
\end{array}\right]
$$

Where 3 is a (possibly empty) vector of variables and $F$ equals $\operatorname{Im}^{-}(f)$ or $\operatorname{Sal}(\lambda y . \phi)$. If 3 is empty, $\operatorname{Sal}(\lambda y . \phi)$ must be chosen as the value of $f$ because there will not be an $\mathrm{Im}^{-}$expression of the appropriate type. Conversely, $\mathrm{Im}^{-}$must be chosen if 3 is not empty. The dots indicate that further parts will be needed to fully analyse 3 .

The specifications of the INCONT and EXCONT are immediatelly seen to conform to the schema for intersective adjectives. Note that the PARTS include $f(\lambda y .2) 3$ if the vector 3 is not empty. ${ }^{19}$ This ascertains that if the expression $F$ on the PARTS list is instantiated as $\mathrm{Im}_{f}^{-[3}$, it is applied to the same variables as $f$, which is just

18 Cf. fn. 2 however.

19 The astute reader may notice that the present exposition does not clarify what exactly a vector of variables is, into which parts it can, if at all, be subdivided and how these are contributed. In fact, I assume 'application of a function to a vector' to mean its successive application to the vector's distinct components. So the vector as such is not an expression at all but treated like one here for the sake of convenience. 
"Different" as a restriction on Skolem functions

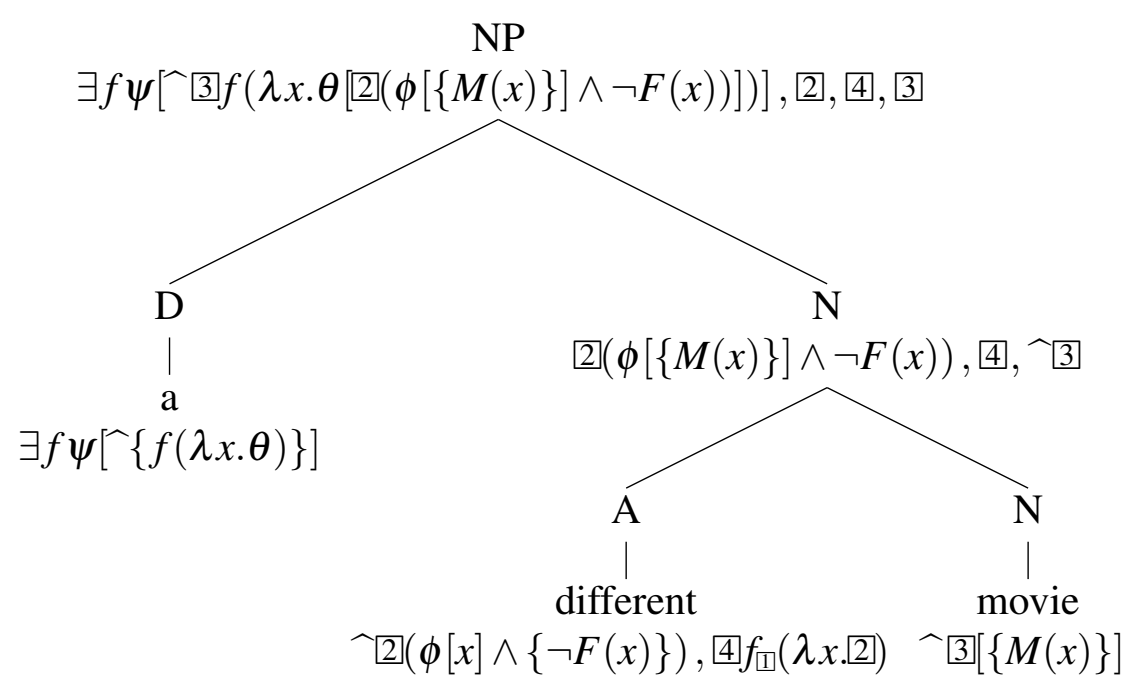

Figure 2 The analysis of a different movie. The most important accumulated parts are notated as comma-separated lists on each node, omitting many of the subparts to avoid unnecessary clutter.

what is needed. 2 refers to the expression $(\phi[y] \wedge 1)$, which is thus placed in the set argument of $f$ as desired.

Crucially, the indefinite article only contributes the part $f(\lambda y .2)$ but not the full $f(\lambda y .2), 3$ (for non-empty 3), which can only be contributed by different. This ascertains that without the occurrence of different or similar items, Skolemisation cannot occur. ${ }^{20}$ The indefinite alone does not provide the resources necessary to apply the function to any individual arguments, which leaves using a pure choice function as the only option if no item like different is available.

Figure 2 shows the derivation of a different movie. It makes use of a convenient notation for LRS: the INCONT value of the annotated node of the tree is enclosed in

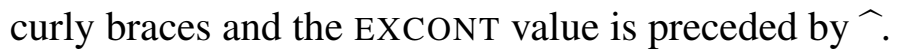

Note how the INCONT and EXCONT values are projected along the head line from the noun movie up to the NP level. This is immediatelly obvious for the INCONT value $M(x)$, but some remarks concerning the EXCONT 3 are in order. It is notated as $3[\{M(x)\}]$ on the movie node, but as $f(\lambda x .2)$ on the NP while both are required to be one and the same expression. The different descriptions serve to highlight the fact that the lexical entry of the noun does not betray what kind of determiner it will combine with. It might turn out to be some quantifier (contributed by, e.g., every) or it might turn out to be a PSCF or an $\imath$ operator. In each of these cases, it will be an expression that contains the INCONT of the noun, and this is how it is represented 
on the noun's node. On the NP level, the SEMANTICS PRINCIPLE will require the identiy of the determiner's EXCONT with that of the noun projection it specifies, and since the determiner's EXCONT is $f(\lambda x . \theta)$, that of the NP, 3 , is then also known to be that.

If this NP occurs in every child watched a different movie and every child contributes a quantifier $\forall y(C(y) \rightarrow \ldots)$ outscoped by $\exists f$, different can be assumed to contribute $f_{y}(\lambda x .2)[\equiv f(\lambda x .2)(y)]$. I.e. $1=y$ and $f$ is thus Skolemised. The EXCONT of the NP will still be $f(\lambda x \cdot 2)$, (a non-head is always required to contribute its EXCONT on its own PARTS list in LRS) and the indefinite article does not contribute its application to a variable. But $f(\lambda x$.2) will have type $\langle e, e\rangle$ and thus cannot serve as an argument to the predicate $W$ of type $\langle e,\langle e, t\rangle\rangle$ contributed by watch. To comply with the constraints on argument linking and supply the proper type, the $f_{y}(\lambda x .2)$ contributed by different must be used, which, being a part, must be used anyway. So the EXCONT (and hence the meaning) of the utterance will come out as (21), just as desired.

$$
\forall y\left(C(y) \rightarrow W\left(y, f_{y}\left(\lambda x \cdot M(x) \wedge \neg \operatorname{Im}_{f}^{-y}(x)\right)\right)\right)
$$

\section{Existential quantification over PSCFs}

So far I have assumed that undefinedness originating from a PSCF being undefined is simply treated like falsity by the existential quantifier that binds the PSCF variable. This view is too simplistic. Consider (22a), which has a reading with a man taking wide and a woman taking narrow scope wrt the negation, as shown in (22b).

$$
\text { a. A man did not invite a woman. }
$$

b. $\exists f \neg \exists g I(f(M), g(W))$

Under the simplistic account given so far, $(22 \mathrm{~b})$ could not fail to be true: choosing any function not defined on $M$ makes $I(f(M), g(W))$ undefined for any value of $g$ and $\exists g \ldots$ will become false under these circumstances. So (22b) itself will become true. To deal with this problem, the existential quantifiers involved are given the following semantics ( $\frac{1}{2}$ stands for undefined $)$ :

$$
\llbracket \exists f_{\tau} \phi \rrbracket^{h}= \begin{cases}1 & \text { iff for some } \alpha \in D_{\tau}, \alpha \text { is a Centered PSCF and } \llbracket \phi \rrbracket^{h[f / \alpha]}=1 \\ \frac{1}{2} & \text { iff for no } \alpha \in D_{\tau}, \llbracket \phi \rrbracket^{h[f / \alpha]} \in\{0,1\} \\ 0 & \text { else }\end{cases}
$$

Syntactically, $\tau$ is required to be a PSCF type.

The first clause should be unsurprising. The second clause, which gives the conditions under which the value of an existentially quantified formula is undefined, requires the value of $\phi$ to be undefined for any value of $f$, whether PSCF or not. 
The else case is of course the conjunction of the negations of both conditions: There must be no PSCF value for $f$ which makes $\phi$ true and some value (PSCF or not) for $f$ must result in an ordinary truth value ( 0 or 1$)$ for $\phi$.

This makes use of the following fact: if $f$ is assigned a value that makes $f(M)$ undefined, $I(f(M), g(W))$ will also be undefined, but it will be so for any possible value of $g$. This informs $\exists g$ that the variable it binds is not the culprit, and so undefinedness is passed over to the negation, which in turn passes it to $\exists f$, where it will not count as verifying the whole sentence (while another value still could).

That the relevant domain of quantification for the undefined-case is all of $D_{\tau}$ and not just the PSCFs of this type accomodates for the possibility of empty set arguments: if there simply are no women (but there is a man) (22a) should be true. But no PSCF can be found in this case that is defined on $W . \exists g \ldots$ would thus be undefined for any value of $f$ and the sentence could never become true. By extending the domain of quantification to all of $D_{\tau}$ it is possible to 'probe' into the scope of the quantifier using functions as values of $g$ that need not return an element of $W$, if anything. Among these a function will be some that return a value to which $f(M)$ either stands or does not stand in the relation $I$, given that $f(M)$ has a value, too. Only if $f(M)$ is undefined will $\exists g \ldots$ also be, which is just the desired result.

\section{Conclusion}

This paper has shown how an analysis of indefinites as Skolemised choice functions can be used to analyse the internal reading of the adjective different under the quantifiers each and every. In sentences like every child watched a different movie, the choice function picks, for each child, something from the set of individuals that are movies and not assigned by the function to any other individual, if such a thing exists. If not, the sentence is false. The sentence is true if every child is assigned a movie she watched. The analysis thus assimilates the semantics of different to that of intersective adjectives, the only difference being that the set of different objects depends, on the internal reading, on the value of a variable bound by every or each. On the external reading of the sentence in question, the set is instead given anaphorically as the set of salient movies, so the external reading is also accounted for. The paper further showed how the semantic ideas outlined can be implemented in an ordinary HPSG grammar, employing Lexical Resource Semantics at the syntax-semantics interface. 


\section{References}

Aloni, Maria. 2001. Quantification under conceptual covers. Amsterdam: University of Amsterdam PhD dissertation. http://maloni.humanities.uva.nl/tesi/tesi.pdf.

Beck, Sigrid. 2000. The semantics of 'different': Comparison operator and relational adjective. Linguistics and Philosophy 23(2). 101-139. doi:10.1023/A:1005566722022.

van Benthem, Johan. 1989. Polyadic quantifiers. Linguistics and Philosophy 12(4). pp. 437-464. doi:10.1007/BF00632472.

Brasoveanu, Adrian. 2011. Sentence-internal 'different' as quantifier-internal anaphora. Linguistics and Philosophy 34(2). 93-168. doi:10.1007/s10988011-9096-3.

Bumford, Dylan \& Chris Barker. 2013. Association with distributivity and the problem of multiple antecedents for singular 'different'. Linguistics and Philosophy 36(5). 355-369. doi:10.1007/s10988-013-9139-z.

Bäuerle, Rainer. 1983. Pragmatisch-semantische Aspekte der NP-Interpretation. In Allgemeine Sprachwissenschaft, Sprachtypologie und Textlinguistik. Festschrift für Peter Hartmann (Tübinger Beiträge zur Linguistik 215), 121-131. Tübingen: Narr.

Carlson, Greg N. 1987. Same and different: Some consequences for syntax and semantics. Linguistics and Philosophy 10(4). 531-565. doi:10.1007/BF00628069.

Geurts, Bart. 2000. Indefinites and choice functions. Linguistic Inquiry 31(4). 731-738. doi:10.1162/002438900554550. https://muse.jhu.edu/article/20032.

Iordăchioaia, Gianina \& Frank Richter. 2015. Negative concord with polyadic quantifiers. Natural Language \& Linguistic Theory 33(2). 607-658. doi:10.1007/s11049-014-9261-9.

Keenan, Edward L. 1992. Beyond the Frege boundary. Linguistics and Philosophy 15(2). 199-221. doi:10.1007/BF00635807.

King, Paul John. 1989. A logical formalism for Head-Driven Phrase Structure Grammar. Manchester: University of Manchester PhD dissertation.

Kratzer, Angelika. 1998. Scope or pseudoscope? Are there wide-scope indefinites? In Susan Rothstein (ed.), Events and Grammar (Studies in Linguistics and Philosophy 70), 163-196. Dordrecht: Springer. doi:10.1007/978-94-011-39694_8.

Levine, Robert, Frank Richter \& Manfred Sailer. (in preparation). Formal semantics: An empirically grounded approach. Unpublished manuscript. Ohio State University and University of Frankfurt a.M.

Penn, Gerald \& Frank Richter. 2004. Lexical Resource Semantics: From theory to implementation. In Stefan Müller (ed.), 11th conference on HeadDriven Phrase Structure Grammar (HPSG), 423-443. Stanford: CSLI Pub- 
"Different" as a restriction on Skolem functions

lications. http://web.stanford.edu/group/cslipublications/cslipublications/HPSG/ 2004/penn-richter.pdf.

Reinhart, Tanya. 1997. Quantifier scope: How labor is divided between QR and choice functions. Linguistics and Philosophy 20(4). 335-397. doi:10.1023/A:1005349801431. http://link.springer.com/article/10.1023/A\% 3A1005349801431.

Richter, Frank. 2004. A mathematical formalism for linguistic theories with an application in Head-Driven Phrase Structure Grammar. Tübingen: University of Tübingen PhD dissertation. https://publikationen.uni-tuebingen.de/xmlui/ bitstream/handle/10900/46230/pdf/richter-diss.pdf.

Sailer, Manfred. 2004. Propositional relative clauses in German. In Stefan Müller (ed.), 11th conference on Head-Driven Phrase Structure Grammar (HPSG), 223-243. Stanford: CSLI Publications. http://web.stanford.edu/group/ cslipublications/cslipublications/HPSG/2004/sailer.pdf.

Schäfer, Roland. 2015. Processing and querying large web corpora with the cow14 architecture. In Piotr Bański, Hanno Biber, Evelyn Breiteneder, Marc Kupietz, Harald Lüngen \& Andreas Witt (eds.), Challenges in the Management of Large Corpora (CMLC-3), 28-34. http://rolandschaefer.net/wp-content/uploads/2015/ 04/RolandSchaefer_COW14_CMLC3.pdf.

Szabó, Zoltán Gendler. 2010. Specific, yet opaque. In Maria Aloni, Harald Bastiaanse, Tikitu de Jager \& Katrin Schulz (eds.), Logic, Language and Meaning (Lecture Notes in Computer Science 6042), 32-41. Berlin; Heidelberg: Springer. doi:10.1007/978-3-642-14287-1_4.

Winter, Yoad. 2001. Flexibility Principles in Boolean Semantics: The Interpretation of Coordination, Plurality, and Scope in Natural Language. (Current Studies in Linguistics 37). Cambridge, MA; London: MIT Press 1st edn.

David Lahm

University of Frankfurt

Norbert-Wollheim-Platz 1

Frankfurt am Main

lahm@uni-frankfurt.de 